\title{
Therapie und Prävention von Problemnarben
}

\author{
Rainer Zumhasch
}

Keloide, hypertrophe und schmerzhafte Narben können physische und psychische Problematiken mit sich bringen. Die Therapie mit Silikon und Kompression hat sich in der Prophylaxe dieser Komplikationen bewährt. Sie sind neben Desensibilisierung und Narbenmassage häufig durchgeführte Behandlungsmaßnahmen. Erfahren Sie, wie sich verschiedene Problemnarben voneinander unterscheiden und worauf Sie bei der Anwendung der einzelnen Behandlungsmaßnahmen achten sollten.

Schlecht verheilte Narben sind ästhetisch sehr störend und können eine funktionelle Behinderung bzw. Funktionseinschränkung an Gelenken nach sich ziehen. Körperliche Narben lassen Betroffene das Trauma nur schwer vergessen. Sie werde unter Umständen somit auch zur seelischen Belastung. Insbesondere Keloide, hypertrophe und schmerzhafte Narben können Probleme hervorrufen. Nur eine gut therapierte, inspektorisch unauffällige Narbe hilft den Betroffenen, die oft schmerzhafte Vergangenheit zu vergessen, und trägt zu uneingeschränkter motorischer Funktion bei.

\section{Merke}

Von Problemnarben sprechen wir beim Vorliegen von Schmerzen, Hypertrophie oder Keloidbildung.

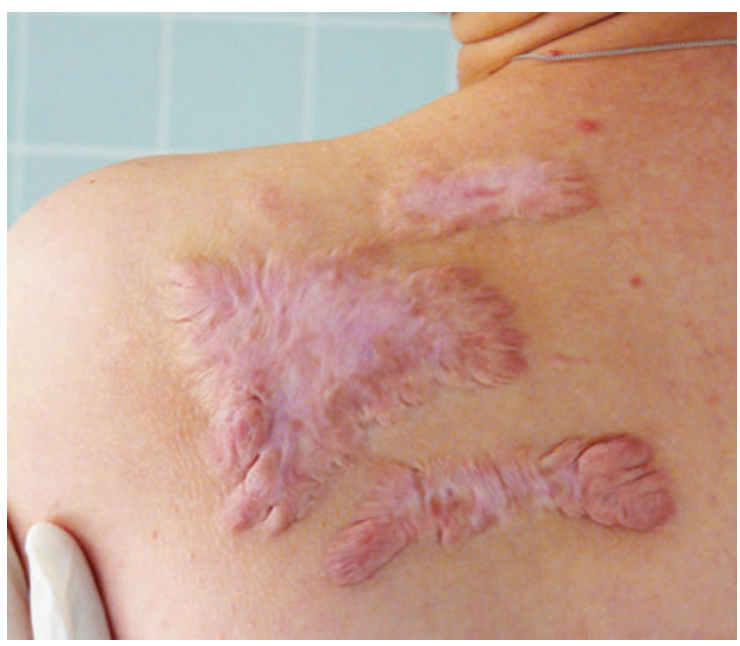

Abb. 1 Flächiges (schweres) Narbenkeloid an der dorsalen Schulter. (Quelle: Zouboulis CC, Weidmann MJ, HarShai Y. Keloidbehandlung mit intraläsionaler Kryochirurgie. Aktuelle Dermatologie 2018; 44(03): 100-108)
Bei ausgeprägten Narben ist eine genetische Disposition gegeben [20]; beispielsweise neigen Afroamerikaner und Chinesen mehr zu Problemnarben als Europäer [16]. Auch Hormone können die Genese von Keloiden und hypertrophen Narben beeinflussen. Auffällig ist, dass schwangere Frauen eher zu einer pathologischen Narbenbildung neigen als Männer [9][22].

\section{Narbenklassifikation}

Ziegler orientiert sich anschaulich an folgender Narbenklassifikation [23]:

Zusammenfassend werden Keloide ( $\triangleright$ Abb. 1 ) als Narben bezeichnet, die (unabhängig von der Genese) über den Rand der ursprünglichen Verletzung hinausragen. Hypertrophe Narben ( $\mathbf{A} \mathbf{A b} \mathbf{b} . \mathbf{2}$ ) hingegen erreichen keine solche Erhabenheit und bleiben auf den Rand der Läsion begrenzt [16].

\section{Therapieoptionen bei Problemnarben}

Es ist grundsätzlich effizienter, Keloide und hypertrophe Narben zu verhindern, als sie im Nachhinein zu behandeln [23]. Die präventive Therapie hat das Ziel, die Gefahr der Ausbildung einer Problemnarbe zu vermindern. Der Übergang auf die eigentliche Behandlung erfolgt dann, wenn tatsächlich eine hypertrophe Narbe oder ein Keloid und nicht eine unreife Narbe diagnostiziert wird. Konzeptionell können sich diverse Therapien und Präventionsschemata, wie beispielsweise das Silikonpad, für beide Narbentypen ähneln. Grundsätzlich gilt: Je früher eine Problemnarbe diagnostiziert wird, umso erfolgreicher ist das zu erwartende Behandlungsergebnis. Eine Epithelisierung sollte vor der Anwendung möglichst stattgefunden haben [23]. 
-Tab. 1 Narbenklassifikation nach Ziegler.

\begin{tabular}{|c|c|}
\hline Einteilung & Beschreibung / Verlauf \\
\hline reife Narbe & helle, flache Narbe \\
\hline unreife Narbe & $\begin{array}{l}\text { Rote, gelegentlich juckende bzw. schmerzhafte Narbe im Verlauf des Umbauprozesses. Viele dieser Narben rei- } \\
\text { fen im Zeitverlauf normal, flachen ab und nehmen eine Pigmentierung ähnlich der umgebenden Haut an. Sie } \\
\text { werden evtl. auch blasser oder etwas dunkler. }\end{array}$ \\
\hline $\begin{array}{l}\text { lineare hypertrophe Narbe } \\
\text { (z. B. operative/ traumatische Narbe) }\end{array}$ & $\begin{array}{l}\text { Rote, erhabene, gelegentlich juckende Narbe, die über den Bereich des ursprünglichen OP-Einschnitts nicht } \\
\text { hinausgeht. Sie entwickelt sich gewöhnlich innerhalb von einigen Wochen nach der Operation. Diese Narben } \\
\text { können sich in drei bis sechs Monaten rasch vergrößern und dann, nach einer stationären Phase, wieder } \\
\text { zurückgehen. Sie reifen generell zu einem erhabenen, leicht strangförmigen Bild mit größerer, variabler Breite. } \\
\text { Der komplette Reifungsprozess kann bis zu zwei Jahre dauern. }\end{array}$ \\
\hline $\begin{array}{l}\text { großflächige hypertrophe Narbe } \\
\text { (z. B. nach Verbrennungen) }\end{array}$ & großflächige rote, erhabene, gelegentlich juckende Narbe, die in den Grenzen der Verbrennungswunde bleibt \\
\hline leichtes Keloid & $\begin{array}{l}\text { Umschriebene erhabene, juckende Narbe, die sich über das normale Gewebe hinaus erstreckt. Sie kann sich bis } \\
\text { zu einem Jahr nach der Verletzung entwickeln und geht nicht von alleine wieder zurück. Einfache chirurgische } \\
\text { Exzision führt oft zum Rezidiv. Der Keloidbildung kann eine genetische Aberration zugrunde liegen (bevorzugte } \\
\text { Stellen sind die Ohrmuscheln). }\end{array}$ \\
\hline schweres Keloid & $\begin{array}{l}\text { Große erhabene Narbe von mehr als } 0,5 \mathrm{~cm} \text {, evtl. schmerzhaft oder juckend, die sich über das normale } \\
\text { Gewebe hinaus ausbreitet. Diese entsteht nach Bagatelltraumen und kann sich über Jahre weiter ausdehnen. }\end{array}$ \\
\hline
\end{tabular}

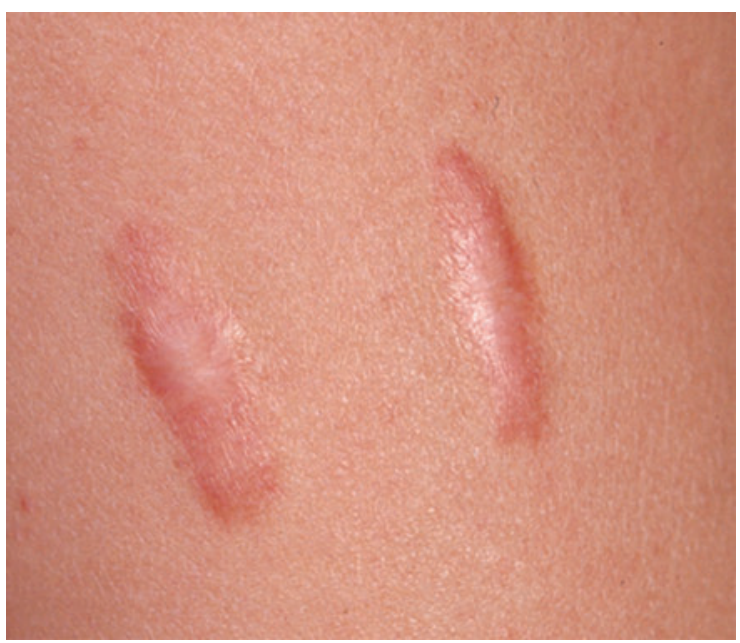

- Abb. 2 Hypertrophe lineare Narben sind erhaben und gehen über den ursprünglichen Schnitt hinaus. (Quelle: Czaika V. Effloreszenzenlehre. In: Neurath F, Lohse A, Hrsg. Checkliste Anamnese und klinische Untersuchung. 5., aktualisierte Auflage. Stuttgart: Thieme; 2018)

Im Folgenden werden die Goldstandards im ergo- und physiotherapeutischen Vorgehen hinsichtlich der Präventiv- bzw. der Problemtherapie aufgezeigt. Je ausgeprägter sich eine Problemnarbe darstellt, desto eher sollten auch ärztliche Interventionen eingebunden werden. Dazu zählen beispielsweise Kortikosteroidinjektionen, Operationen, Lasertherapie, Stickstofftherapie und das Microneedling.

\section{Silikonfolien und Silikongel}

Prävention und Problemtherapie mit Silikonfolien oder -gel sind der therapeutische Goldstandard in der
Behandlung von Keloiden und hypertrophen Narben ( Abb. 3). Ihre Wirkung ist in mehr als 27 Studien nachgewiesen [13]. Die Therapie sollte frühestmöglich begonnen werden [16], erfahrungsgemäß nachdem die Fäden gezogen wurden. Ein Autor verwendete die Silikonfolien bereits 24 Stunden postoperativ [6][16].

Sowohl bei frischen als auch bei alten Narben zeigten Silikonfolien und -gels ein Abflachen des hypertrophen Gewebes [17][19]. Zudem scheinen die Folien die Ausbildung von Kontrakturen und die Schrumpfung von Trans-

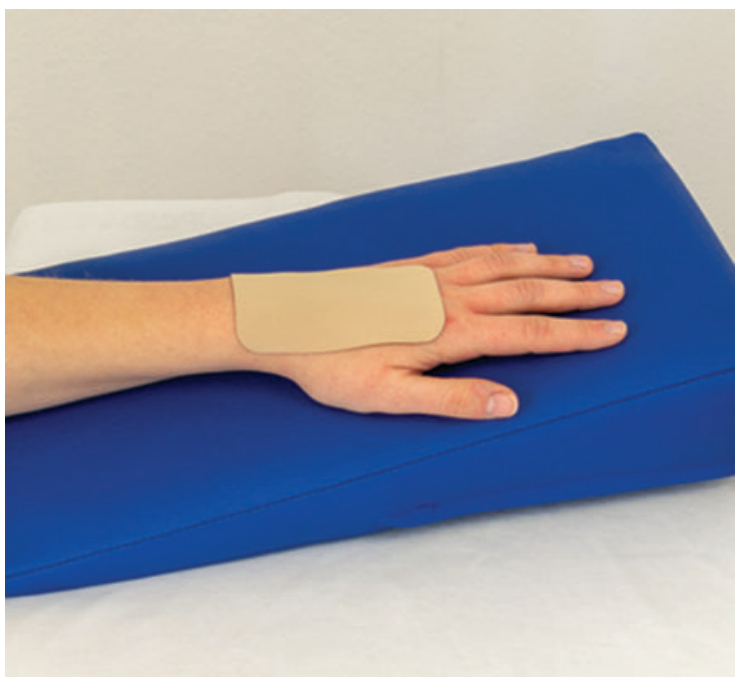

- Abb. 3 Silikonfolien können ab Fadenzug zur Therapie von problematischen Narben zum Einsatz kommen. Silikongel eignet sich auch für Stellen, die durch viel Beweglichkeit oder besonders kleine Fläche ungünstig für Folien sind (Quelle: R. Zumhasch) 
plantaten (beispielsweise bei kleinen bis mittleren Brandwunden) zu verhindern [7][12]. Auch wurden die Narben weicher und ihre Elastizität nahm im Laufe der Applikation zu [16]. Des Weiteren konnte bei einigen Patienten eine Schmerzreduktion nach ein bis zwei Stunden beobachtet werden [7]. Die exakten Wirkmechanismen sind bis dato noch nicht geklärt. Es wird eine verstärkte Rehydrierung im Narbenareal diskutiert, die möglicherweise einer überschießenden Neovaskularisierung bzw. kapillaren Hyperämie sowie den damit verbundenen und überschießenden Kollagenablagerungen entgegenwirkt [23].

Der durch das Silikon ausgeübte Druck hat keine ausreichende therapeutische Wirkung. Um einen Erfolg über Kompression zu erreichen, sind Drücke zwischen 15 und $40 \mathrm{mmHg}$ [11] über eine Applikationsdauer von mindestens vier bis sechs Monaten, bei täglicher Anwendung von mind. 23 Stunden nötig. Unter der Silikonfolie entstehen aber nur Drücke zwischen 1 und 12,8 mmHg [1].

Silikonfolien sollten mindestens zwölf Stunden täglich, erfahrungsgemäß bis zu 23 Stunden über einen Zeitraum von mindestens zwei Monaten bis zu einem Jahr

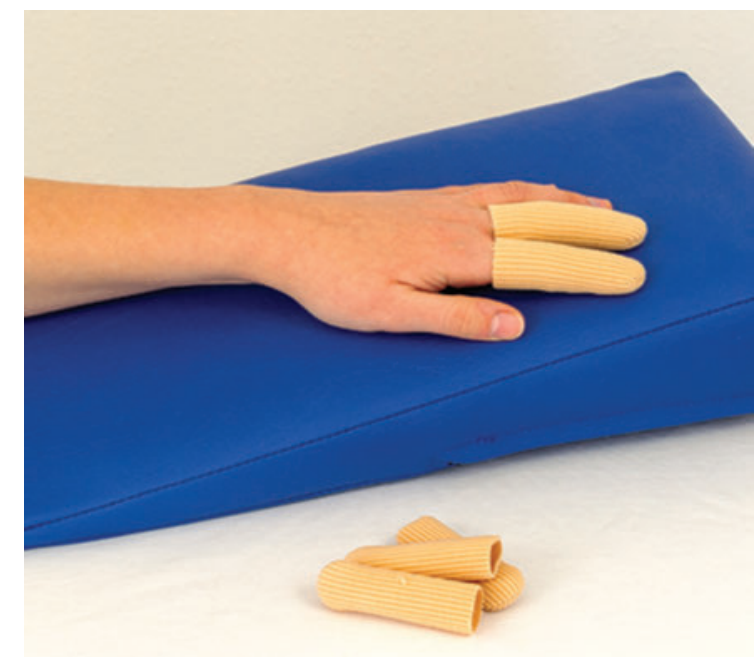

Abb. 4 Digi-Sleeves eignen sich zur Kompressionstherapie von Fingernarben. (Quelle: R. Zumhasch)

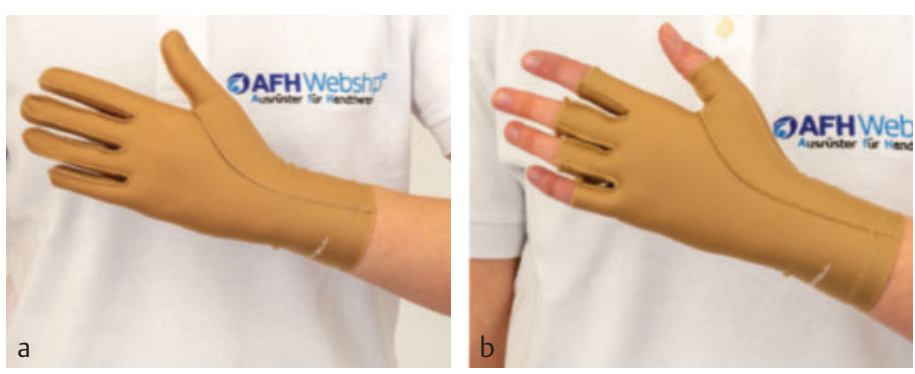

Abb. 5 a, b Kompressionshandschuh mit und ohne Fingerspitzeneinschluss. (Quelle: R. Zumhasch) getragen werden [20]. Nachteilig ist die Kurzlebigkeit der einzelnen Folien bzw. des Gels von ca. drei bis sechs Wochen [16] und die damit verbundene hohe Kostenintensität. Empfehlenswert sind Produkte der Marken ScarFX und RENOSIGEL.

\section{Kompressionstherapie}

Die Kompressionstherapie ist der Goldstandard, insbesondere bei hypertrophen Verbrennungsnarben [4][18][21]. Empfehlenswert sind Drücke zwischen $24 \mathrm{mmHg}$ bis $30 \mathrm{mmHg}$, bei einer Applikationsdauer von mindestens sechs bis zwölf Monaten [11]. Die Wirkung liegt in der verminderten Blutzufuhr und der damit verbundenen Sauerstoffreduktion unter der applizierten Kompression. Da die Kollagensynthese sauerstoffabhängig ist, die Lyse hingegen nicht, vermindert die Druckapplikation die Synthese bei gleichzeitigem Fortschreiten der Lyse [8].

Die Kompression hemmt nicht nur die Bildung hypertropher und kontrakter Narben, sondern unterstützt auch die Ödemreduktion [20]. An den Fingern eignen sich sogenannte Digi-Sleeves ( $>$ Abb.4). In konkaven Aushöhlungen wie der Hohlhand können zur Kompressionstherapie Zweikomponentenmassen als Inlay für Orthesen genutzt werden [10]. Dabei handelt es sich um Elastomere, also formfeste, aber elastisch verformbare Kunststoffe, beispielsweise von der Marke Erkodent. An der Hand kommen Konfektionsdruckhandschuhe ( $\mathbf{A b b}$. 5) mit oder ohne Fingerspitzeneinschluss (z. B. die Marken EDEMA und Jobst) oder Maßhandschuhe, welche regelmäßig angepasst werden müssen, zum Einsatz [15].

\section{Tapestreifen}

Zwei Studien beschreiben einen positiven Effekt der postoperativen Applikation (nach dem Fädenziehen) von hautfreundlichen Tapestreifen über mehrere Wochen [3][14]. Die Wirkung ist nicht geklärt; möglicherweise sind hier die mechanischen Effekte (Drucktherapie) sowie die okklusiven Aspekte (Silikontherapie) anzuführen [23].

\section{Desensibilisierung}

Patienten mit einer Hypersensibilität empfinden infolge eines normalen, nicht schmerzhaften Reizes eine übersteigerte, unangenehme und oft auch schmerzhafte Reaktion [2]. Therapeutisches Ziel ist es, die Toleranz des Patienten für Berührungen in den überempfindlichen Gebieten zu steigern bzw. das physiologische Empfinden anzupassen. Dadurch soll eine größtmögliche Funktionalität im Alltag erreicht werden [2]. Das Training der Desensibilisierung kommt nach Amputationen, Neuromen bzw. peripheren Nervenverletzungen und insbesondere bei hypersensiblen Narben zur Anwendung. Je früher ein solches Training appliziert wird, desto erfolgreicher ist die Therapie [5]. Drei bis fünf Wiederholungen über einen Zeitraum von ca. fünf bis dreißig Minuten sind empfehlenswert; der Patient sollte die Intervention 


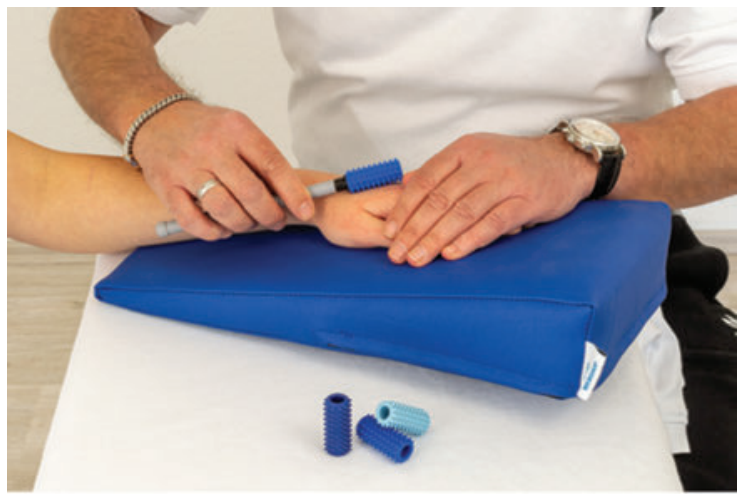

- Abb. 6 Der „Dermoroller“ desensibilisiert und massiert das Narbengewebe. (Quelle: R. Zumhasch)

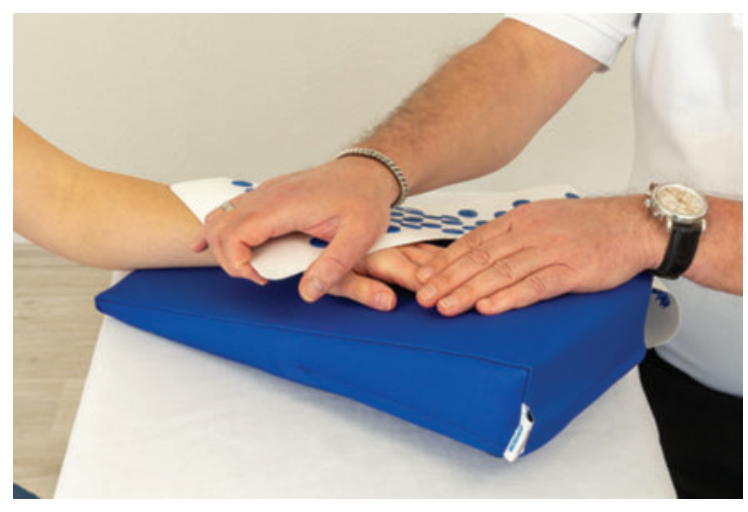

Abb. 7 Die Nadelreizmatte kann in der letzten Phase des Desensibilisierungstrainings Anwendung finden. (Quelle: R. Zumhasch)

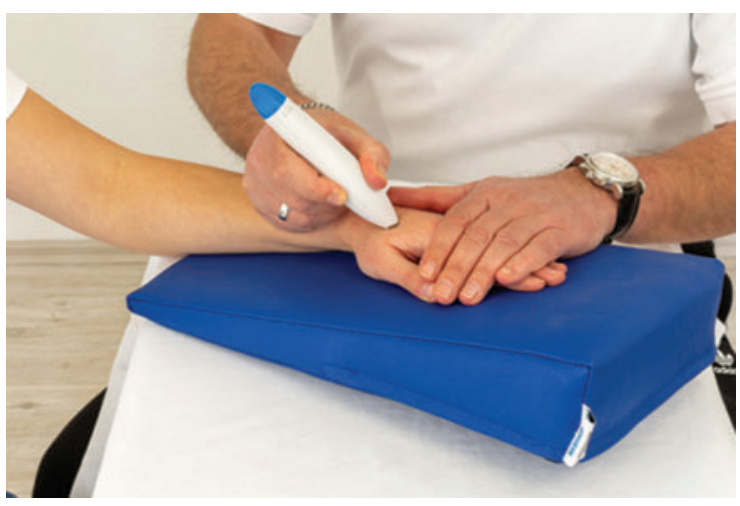

- Abb. 8 Vibrationsreize sprechen vor allem die VaterPacini-Körperchen in der Dermis an. (Quelle: R. Zumhasch) konzentriert und motiviert durchführen [2]. Je nach Befund werden die betroffenen Hautareale in starke und weniger starke hypersensible Areale gegliedert. Des Weiteren muss der Stimulus mit den geringsten Irritationen eruiert werden, mit dessen Reiz letztendlich der Desensibilisierungsprozess bzw. das Abhärtungstraining der Narbe einzuleiten ist. Die Stelle mit der größten Toleranz gilt als Ausgangsstellung, bis der adaptierte Reiz als angenehm empfunden wird. Es können Reize vom Wattebausch oder von Wolle bis hin zur Zahnbürste bzw. zu einem extra dafür konzipierten „Dermoroller“ mit vier unterschiedlichen Härtegraden verwendet werden ( A Abb. 6). Vorteil des „Dermorollers“ ist, dass er neben der desensibilisierenden Wirkung auf die Drucksensoren der Oberhaut (Merkel-Tastscheiben und den MeissnerKörperchen) auch einen Massageeffekt aufweisen kann und somit weiterlaufenden Verwachsungen im Narbengewebe vorbeugt. Mit Erreichen des stärksten Widerstands kann bei großflächigen Arealen auch eine Nadelreizmatte Verwendung finden ( $\triangleright$ Abb.7). Durch den Massageeffekt eines Vibrationsgerätes kann im Weiteren auch Irritationen der Vibrationsrezeptoren in der Lederhaut (Vater-Pacini-Körperchen) entgegengewirkt werden ( $\triangleright$ Abb. 8).

\section{Fazit}

Je früher bei Problemnarben therapeutisch interveniert wird, umso größer ist der Effekt bei kleinstmöglichem Einsatz von risikoarmen therapeutischen Techniken. Dazu zählen Silikon- und Kompressionsapplikation, die Anwendung von Tapestreifen und die Desensibilisierung. Weiterhin können diverse Techniken wie die Narbenmassage, das Schröpfen und motorische Übungsprogramme mit ins therapeutische Vorgehen integriert werden, um möglichen Verwachsungen frühzeitig vorzubeugen. Ziel ist die Wiederherstellung der physiologischen Funktionen.

\section{Autorinnen / Autoren}

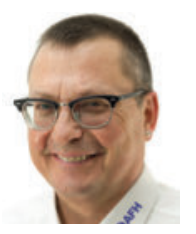

\section{Rainer Zumhasch}

Anerkannter Lehrtherapeut im Bereich Handtherapie sowie Referent an verschiedenen Fachkongressen, langjährige eigene Praxistätigkeit, regelmäßige nationale wie internationale Publikationen in Fachzeitungen und Fachbüchern, redaktionelle Arbeit, Produktentwickler und -berater für diverse Firmen und Geschäftsführer des AFH-Webshops

Korrespondenzadresse

Rainer Zumhasch

AFH-Webshop

Pyrmonter Straße 50

32676 Lügde

E-Mail: r.zumhasch@premium-therapie.de 
Literatur

[1] Ahn ST, Monafo WW, Mustoe TA. Topical silicone gel: A new treatment for hypertrophic scars. Surgery 1989; 106: 781787

[2] Bollinger Herzka T. Therapieprozess. In: Koesling C, Bollinger Herzka T, Hrsg. Ergotherapie in der Orthopädie, Traumatologie und Rheumatologie. Stuttgart: Thieme; 2008

[3] Davery RB, Wallis KA, Bowering K. Adhesive contact median: An update of graft fixation and burn scar managment. Burns 1991; 17: 313

[4] Fricke NB, Omnell ML, Dutscher KA et al. Skeletal and dental disturbances in children after facial burns and pressure garment use: A 4-year follow-up. J Burn Care Rehabil 1999; 20: 239

[5] Frykmann GK, Waylett J. Desensitization. Handrehabilitation: A practical guide. New York: Churchill Livingstone; 1993

[6] Gold MHMD. A controlled clinical trial of topical silicone gel sheeting in the treatment of hypertrophic scars and keloids. J Am Acad Dermatol 1994; 30: 506-507

[7] Gold MHMD. Topical silicone gel sheeting in the treatment of hypertrophic scars and keloids. J Dermatol Surg Oncol 1993; 19: 912-916

[8] Hardy M, Woodall W. Therapeutic effects of heat, cold and stretch on connective tissue. J Hand Ther 1998; 11: 148-156

[9] Kelly PA. Keloids. Dermatology Clinics 1988; 6: 413-424

[10] Knaus W. Narbenbehandlung in der Ergotherapie. Praxis Ergotherapie 2012; 25(2): 64-69

[11] Niessen FB, Spauwen PHM, Schalkwijk J, Kon M. On the nature of hypertrophic scars and keloids: A review. Plast Reconstr Surg 1999; 104: 1435
[12] Perkins K, Davey RB, Wallis KA. Silicone gel: A new treatment for burn scars and contractures. Burns 1982. 9: 201-228

[13] Poston J. The use of silicone gel sheeting in the management of hyperthrophic and keloid scars. J Wound Care 2000; 9: 10

[14] Reiffel RS. Prevention of hypertrophic scars by longterm paper tape application. Plast Reconstr Surg 1995; 96: 1715

[15] Schröder B. Handtherapie. Erweiterte und überarbeitete Auflage. Stuttgart: Thieme; 2008

[16] Schuster K. Untersuchungen zur Wirksamkeit der Behandlung von Keloiden bzw. hypertrophen Narben in vivo mit intraläsionalem Triamcinolonacetonid bzw. Silikon-Gel Folie [Dissertation]. München: Technischen Universität München; 2001: 6ff.

[17] Sebastian G, Buxbaum-Conradi H, Fischer S et al. Wirksamkeit und Verträglichkeit von Silikon-Gel bei der Behandlung verschiedener Narben (Anwendungsbeobachtung mit Dermatix ${ }^{\mathrm{TM}}$ ). Akt Dermatol 2004; 30-78

[18] Tredget EE. Management of the acutely burned upper extremity. Hand Clin 2000; 16: 187

[19] Van den Kerckhove E, Boeckx W, Kochuyt A. Silicone patches as a supplement for pressure therapy to control hypertrophic scarring. J Burn Care Rehabil 1991; 12: 361-369

[20] Waldner-Nilsson B. Wund- und Narbenbehandlung. In: DidayNolle, Breier S, Slatosch Wintsch DU, Reiter Eigenheer A, Hrsg. Handrehabilitation, Band 1 Grundlagen, Erkrankungen. Berlin Heidelberg: Springer; 2009

[21] Ward RS. Pressure therapy for the control of hypertrophic scar formation after burn injury: A history and review. J Burn Care Rehabil 1991; 12: 257

[22] William J, Sahl JR, Clever H. Review: Cutaneous scars: Part I. Int J of Dermatol 1994; 33: 681-691

[23] Ziegler UE. Internationale Empfehlungen zur Narbenbehandlung. Zentralbl Chir 2004; 129: 296-306 